\title{
E4F1: a novel candidate factor for mediating BMI1 function in primitive hematopoietic cells
}

\author{
Jalila Chagraoui, ${ }^{1}$ Sherry L. Niessen, ${ }_{1}^{1}$ Julie Lessard, ${ }^{1}$ Simon Girard, ${ }^{1}$ Philippe Coulombe, ${ }^{2}$ \\ Martin Sauvageau, ${ }^{1}$ Sylvain Meloche, ${ }^{2,3}$ and Guy Sauvageau ${ }^{1,4,5}$ \\ ${ }^{1}$ Laboratory of Molecular Genetics of Hematopoietic Stem Cells, Institut de Recherche en Immunologie et Cancérologie \\ (IRIC), C.P. 6128 succursale Centre-Ville, Montréal, Québec H3C 3J7, Canada; ${ }^{2}$ Laboratory of Signaling and Cell Growth, \\ Institut de Recherche en Immunologie et Cancérologie (IRIC), C.P. 6128 succursale Centre-Ville, Montréal, Québec H3C \\ 3J7, Canada; ${ }^{3}$ Departments of Molecular Biology and Pharmacology, Université de Montréal, Montréal, Québec, Canada; \\ ${ }^{4}$ Department of Medicine, Division of Hematology, and Leukemia Cell Bank of Quebec, Maisonneuve-Rosemont Hospital, \\ Montréal, Québec H1T, 2M4, Canada
}

The Polycomb group gene Bmi1 is essential for the proliferation of neural and hematopoietic stem cells. Much remains to be learned about the pathways involved in the severe hematopoietic phenotype observed in Bmi1 homozygous mutant mice except for the fact that loss of $p 53$ or concomitant loss of $p 16^{\text {Ink4a }}$ and $p 19^{\text {Arf }}$ functions achieves only a partial rescue. Here we report the identification of E4F1, an inhibitor of cellular proliferation, as a novel BMI1-interacting partner in hematopoietic cells. We provide evidence that Bmi1 and E4f1 genetically interact in the hematopoietic compartment to regulate cellular proliferation. Most importantly, we demonstrate that reduction of $E 4 f 1$ levels through RNA interference mediated knockdown is sufficient to rescue the clonogenic and repopulating ability of $\mathrm{Bmi1}^{-/-}$hematopoietic cells up to 3 mo post-transplantation. Using cell lines and MEF, we also demonstrate that INK4A/ARF and p53 are not essential for functional interaction between Bmi1 and E4f1. Together, these findings identify E4F1 as a key modulator of BMI1 activity in primitive hematopoietic cells.

[Keywords: Polycomb; Bmi1; hematopoietic stem cell self-renewal; E4F1; senescence]

Supplemental material is available at http://www.genesdev.org.

Received January 25, 2006; revised version accepted June 6, 2006.

The Polycomb group (PcG) proteins were initially identified in Drosophila as repressors of homeotic genes (Moehrle and Paro 1994). PcG proteins assemble into discrete chromatin-associated complexes that ensure the stable inheritance of gene expression state through cell division. The Polycomb repressive complex 1 (PRC1) includes BMI1, RING1/2, MEL-18, RAE28/MPH1, and M33/CBX2, while other complexes (PRC2/3/4) comprise, among other proteins, EED, EZH1/2, and SUZ12 (Valk-Lingbeek et al. 2004). Evidence that the molecular activity of PRC results in covalent modifications of both nucleosomal and nonnucleosomal histones is rapidly accumulating (Wang et al. 2004; Cao et al. 2005; Hernandez-Munoz et al. 2005). The SET domain proteins $\mathrm{EZH1} / 2$ are methyltransferases that specifically target lysine 27 in histone $\mathrm{H} 3$ (H3K27) and lysine 26 in histone $\mathrm{H} 1$ (H1K26). Trimethylation of $\mathrm{H} 3 \mathrm{~K} 27$ is required for recruitment of the BMI-containing PRC1 complex, in

${ }^{5}$ Corresponding author.

E-MAIL guy.sauvageau@umontreal.ca; FAX (514) 343-7379.

Article is online at http://www.genesdev.org/cgi/doi/10.1101/gad.1453406. which BMI1 represents an essential cofactor of the RING1/2 monoubiquitin E3 ligase implicated in the ubiquitination of histone H2A (Cao et al. 2005).

PcG genes regulate the activity of many different types of blood cells. These include the T- and B-cells whose maturation and/or proliferation is dependent on $\mathrm{Mel}-18$, Bmi1, Rae-28/Mph1, and M33/Cbx2 genes /Core et al. 1997; Tokimasa et al. 2001; Ohta et al. 2002; Lessard and Sauvageau 2003; Miyazaki et al. 2005). Hematopoietic stem cells (HSCs), which possess the unique property to generate all blood cell types and to self-renew, are also highly dependent on the activity of several PcG proteins. The role for BMI1 in HSC proliferation and self-renewal is best characterized. Bmil expression is mostly detected in primitive human $\left(\mathrm{CD} 34^{+} \mathrm{CD} 45^{-} \mathrm{CD} 71^{-}\right)$and mouse $\left(\mathrm{Scal}^{+} \mathrm{Lin}^{-}\right)$bone marrow (BM) cells (Lessard et al. 1998; Lessard and Sauvageau 2003; Park et al. 2003). Nullizygosity for the Bmil gene in mice leads to severe aplastic anemia presumably due to a progressive impairment of HSC self-renewal (van der Lugt et al. 1994; Lessard et al. 1999; Lessard and Sauvageau 2003; Park et al. 2003). Retroviral expression of $B \mathrm{mil}$ in $\mathrm{Bmil}^{-/-}$fetal liver (FL) cells 
completely rescued the proliferative activity of myeloid progenitors to wild-type levels, indicating that this gene is dispensable for the generation of FL-derived myeloid progenitors but essential for their full proliferative activity (Lessard and Sauvageau 2003). In line with these results, complementation studies suggest that HSC numbers are normal (A. Faubert and G. Sauvageau, in prep.) in embryonic day 14.5 (E14.5) Bmi1-null FLs. Moreover, studies by Park and colleagues (Park et al. 2003) documented the inability of BM and FL-derived $\mathrm{Bmi1}^{-/-}$cells to sustain long-term production of blood cells in transplantation chimers, demonstrating a cell-autonomous impairment of their self-renewal potential.

The proliferative potential of $\mathrm{Bmi1}^{-/-}$hematopoietic progenitors is gradually lost from fetal to adult developmental stages, possibly indicating that these cells undergo a premature senescence (van der Lugt et al. 1994; Lessard et al. 1999; Lessard and Sauvageau 2003). Supporting this possibility, Bmi1/- hematopoietic progenitors accumulate high levels of the senescence marker SA- $\beta$-gal (see Results) upon short-term proliferation. The senescent phenotype is also associated with increased levels of the tumor suppressor proteins $\mathrm{p} 16^{\mathrm{INK} 4 \mathrm{~A}}$ and p19 ${ }^{\mathrm{ARF}}$, which are known to impair CDK4- and CDK6induced phosphorylation of $\mathrm{Rb}$ and prevent the functional inactivation of p53 by binding to MDM2, respectively (Serrano et al. 1993; Quelle et al. 1995; Kamijo et al. 1998; Pomerantz et al. 1998; Weber et al. 1999). In the absence of Bmi1, primary mouse embryonic fibroblasts (MEFs) are impaired in progression into the $S$ phase of the cell cycle and undergo premature senescence (Jacobs et al. 1999). In these fibroblasts and in Bmi1-deficient lymphocytes, the expression of the tumor suppressors $p 16^{I n k 4 a}$ and $p 19^{A r f}$ is raised markedly. Conversely, overexpression of Bmi1 in MEFs clearly down-regulates the expression of $p 16^{\text {Ink4a }}$ and $p 19^{A r f}$, delays replicative senescence, and facilitates immortalization (Jacobs et al. 1999). Similarly, overexpression of Bmi1 in primary human fibroblasts extended their replicative life span by suppressing the $\mathrm{p} 16^{\mathrm{INK} 4 \mathrm{~A}}$-dependent senescence pathway (Itahana et al. 2003).

Iwama and collaborators (Iwama et al. 2004) showed that primitive (i.e., lineage-negative) $\mathrm{Bmi1}^{-/-} \mathrm{E} 14.5 \mathrm{FL}$ cells express high levels of $p 16^{\text {Ink } 4 a}$ and $p 19^{A r f}$ compared to wild-type controls, whereas only $p 16^{\text {Ink } 4 a}$ levels were high in total (unpurified) FL cells. Although not investigated in primitive hematopoietic cells until recently, inactivation of the INK4A/ARF pathway marginally reduced the hematopoietic cell proliferation defects of Bmi1-null mutants (Bruggeman et al. 2005; Molofsky et al. 2005), indicating that INK4A/ARF is not the only critical in vivo downstream target for BMI1 in the regulation of HSC activity. In support to this, we reported that $\mathrm{Bmi1}^{-/-}$leukemia cell lines lacking expression of $p 16^{\text {Ink } 4 a}$ and $p 19^{A r f}$ depended on ectopic expression of Bmil for their ability to produce acute leukemia in vivo (Lessard and Sauvageau 2003).

Thus, the identification of factors that mediate BMI1 function in HSCs is of significant interest. In this study, we show that the inhibitor of cell proliferation E4F1 in- teracts physically and genetically with Bmil to regulate HSC activity.

\section{Results}

\section{Identification of the BMI1-interacting protein E4F1}

A yeast two-hybrid assay was employed using BMI1 as "bait" to screen a human fetal liver-derived cDNA library (enriched for primitive hematopoietic cells). From a total complexity of $\sim 1$ million cotransformants, 14 nonauxotrophic clones representing eight different genes were identified. One interesting clone corresponded to the last four C-terminal zinc finger domains (amino acids 346-783) (Fig. 1A) of the E1A-regulated transcription factor, E4F1 (Fig. 1B, sections 2 and 6). The interaction between BMI1 and E4F1 was further suggested using pull-down assays performed with in vitro translated proteins (Fig. 1C, lane 1). The interaction surface between BMI1 and E4F1 was mapped using the yeast two-hybrid system and involved the central Helix-Turn-Helix (HTH) domain of BMIl and the last four C-terminal zinc fingers domains of E4F1 (Fig. 1D,E).

To determine whether BMI1 and E4F1 associate in vivo in mammalian cells, coimmunoprecipitations were carried out first using 293T cells transfected with HAtagged E4F1. Western blot analysis of proteins coimmunoprecipitated with BMI1 confirmed the interaction between BMI1 and tagged E4F1 (Fig. 1F). We also analyzed the subcellular distribution of the complex; interestingly, we showed that the endogenous E4F1 (see asterisk in Fig. 1F, lane 2) as well as the tagged protein (Fig. 1F, lane 4) were complexed to BMIl specifically in the cytoplasmic compartment (Fig. 1G). The subcellular localization of endogenous BMI1 and E4F1 was confirmed using confocal microscopy (data not shown).

\section{Bmil interacts genetically with E4f1 in cell lines}

To establish further the functional relationships between Bmi1 and E4f1, we first examined the impact of overexpressing Bmi1 and E4f1 on the proliferation of NIH 3 T3 cells (Fig. 2A,B). While E4f1 and Bmi1 showed opposite effects on the proliferation rate of these cells, Bmi1 overexpression partially but consistently rescued the proliferative defect induced by E4f1. Moreover, we observed that the HTH domain of BMI1, through which E4F1 binds, is required to abrogate the proliferation defect associated with E4F1 (Fig. 2A), while the $\triangle$ PEST mutant, which still associates with E4F1, provides a rescue similar to that observed with wild-type BMI1 (data not shown).

Changes in proliferation rates were accompanied by modification of $\mathrm{Rb}$ phosphorylation status. Indeed, ectopic expression of E4f1 resulted in accumulation of the hypophosphorylated form of $\mathrm{Rb}$ (hypo- $\mathrm{Rb}$ ), and reintroduction of Bmi1, but not the $\triangle \mathrm{HTH}$ mutant, prevented this accumulation (Fig. 2C).

To further analyze the genetic interaction between 
Figure 1. Identification of the BMI1 interacting protein E4F1. (A) Schematic representation of $E 4 f 1$ and the isolated clone C-E4f1. (B) BMI1 specifically associates with E4F1 in yeast. AH109 yeast were cotransfected with GAL4-DNA binding (pGBKT7) and GAL4-transactivation domain (pGADT7 or pACT2) expression vectors encoding Bmi1, E4f1, Ring $1 b$ (positive control), and Large $\mathrm{T}$ antigen or Lamin $\mathrm{C}$ (both negative controls). Interactions were monitored between (1) BMI1 and Large T, $(2,6)$ BMI1 and C-E4F1, (3) BMI1 and GAL4 $\mathrm{AD},(4)$ GAL4 alone (5) C-E4F1 and Lamin C, (7) C-E4F1 and GAL4 DB, and (8) BMI1 and RING1B (positive control). $(C)$ Direct physical association between BMIl and E4F1. In vitro transcribed-translated ${ }^{35} \mathrm{~S}$ labeled proteins were immunoprecipitated (IP) with an antibody specific to Bmil and separated by SDS-PAGE. $(D, E)$ Interaction domain between BMI1 and E4F1 assessed in yeast. The correct folding of the Bmil deletion mutants was ensured by demonstrating their potential to interact with RING1B and HPH1, proteins known to interact with the RING and HTH domains of BMI1, respectively. (F) BMI1 and E4F1 specifically coimmunoprecipitate in mammalian cells. $293 \mathrm{~T}$ cells were transfected with HA-HOXB4 (negative control), HA-RING1B (positive control), or an HA-tagged version of the C-terminal E4F1 fragment clone isolated in the screen. Immunoprecipitations were performed overnight on total cellular extracts using an antibody specific to BMI1. Western blots were revealed using anti-HA antibody. $(G)$ BMI1 and E4F1 associate in the cytoplasm. Nuclear (Nuc) and cytoplasmic (Cyto) extracts were generated from HA-E4F1 and control K562 cells. Extracts were immunoprecipitated with an antibody to BMI1, and associated proteins were revealed by Western blotting with antibodies to HA, E4F1, and BMI1. ( ${ }^{\star}$, lane 2 ) Endogenous E4F1.

Bmi1 and E4f1, we generated shRNA vectors that efficiently suppressed Bmi1 and E4f1 expression (Fig. 2D). Knockdown of Bmil severely impaired proliferation of NIH 3 T3 cells, while the reduction of E4F1 protein levels enhanced cellular proliferation. Importantly, the proliferative defect induced by Bmi1 knockdown was largely rescued by the reduction in E4F1 protein levels (Fig. 2E).

\section{Bmil/E4f1 genetic interaction in the absence of $\mathrm{p} 19^{\text {Arf }}$} and $\mathrm{p} 53$

Several experiments were performed to determine whether the $p 19^{A r f}-p 53$ axis is essential for E4F1-mediated effects. The proliferation-inhibitory effect of E4f1 overexpression was first examined in various cell lines carrying defined genetic alterations in the $p 19^{A r f}$ or/and p53 genes. Results from these studies suggested that INK4A/ARF is not required for E4F1-induced proliferation defect, but a correlation was observed between the responses to E4f1 overexpression and the p53 status (Supplemental Table 1). We next studied the effect of E4F1 on proliferation of the p53-deficient K562 cells, which appeared to be refractory to E4F1-mediated proliferation arrest (Fig. 3A). Interestingly, reintroduction of p53 inhibited expansion of these cells without restoring the proliferation-inhibitory function of E4F1 (Fig. 3A), indicating either that additional factors or p53-confounding factors are involved.

To test this possibility further, the human papilloma virus E6 gene was ectopically expressed in E4f1-overexpressing NIH $3 \mathrm{~T} 3$ cells (INK4A/ARF locus deleted) to acutely induce p53 degradation, thereby changing their ability to proliferate (Fig. 3B). Interestingly, E6-transduced NIH 3 T3 cells remained sensitive to E4F1-induced proliferation-inhibitory function (Fig. 3B). MEFs were then isolated from $p 53^{-/-}$mice and from littermate controls (e.g., $p 53^{+/+}$), and the presence of Bmi1-E4f1 genetic interaction was evaluated. The results presented in Figure $3 \mathrm{C}$ indicate that $p 53^{-/-}$MEFs show a reduction in BrdU incorporation in response to shRNA to Bmil and a strong increase in response to shE4f1. Moreover, the genetic interaction between $E 4 f 1$ and Bmi1 is conserved in these $p 53^{-/-}$cells. Importantly, MEFs from these experiments were also assessed for apoptosis by Annexin V and PI staining and, as observed in NIH 3 T3 cells, no difference was detected between the various populations (data not shown).

We next tested the p53-mediated induction of p21 in irradiated NIH 3T3 cells engineered to express high levels of E4F1 (Fig. 3D) and found that p21 induction in 
A

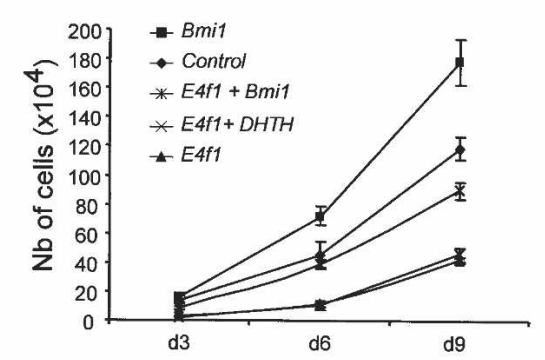

D

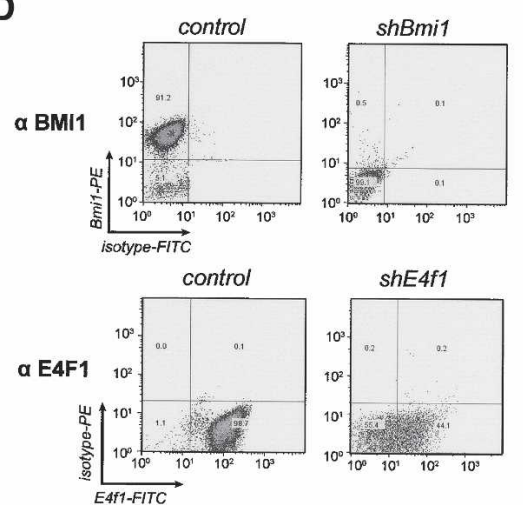

B

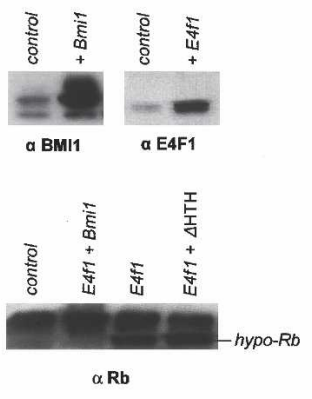

E

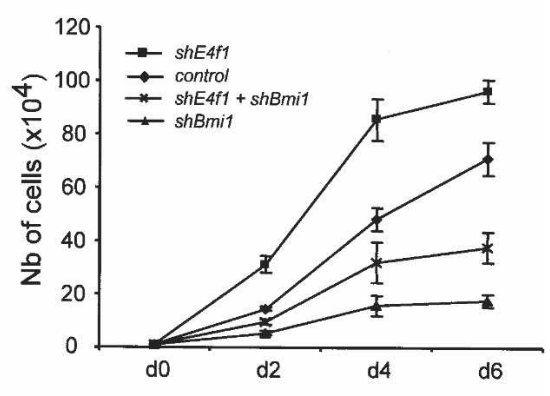

Figure 2. Genetic interaction between Bmi1 and E4f1 in NIH 3T3. (A) NIH 3T3 cells that express E4f1 or Bmi1, or coexpress E4f1 and Bmi1 were plated in triplicate, and counted at the indicated time points. Cells were collected at day 9 and analyzed by Western blot for the expression of $(B)$ BMI1 and E4F1 or $(C) \mathrm{Rb}$ protein (hypo-Rb:hypophosphorylated $\mathrm{Rb}$ ). (D) NIH 3T3 cells infected with retroviruses encoding shBmi1, shE4f1, or an empty vector were fixed, permeabilized, and stained sequentially with primary antibodies (mouse anti-BMI1 and rabbit antiE4F1) and the matched conjugated secondary antibodies (anti-mouse PE and antirabbit FITC) and then analyzed by flow cytometry. (E) Proliferation of NIH 3T3 cells infected with retrovirus encoding shBmi1, shE4f1, or both. For $A$ and $E$, proliferation was measured in three independent experiments each resulting from newly infected and selected cellular populations assessed in duplicates within $6 \mathrm{~d}$ of infection. Data are given as means $\pm \mathrm{SD}$. Populations assessed in these experiments are polyclonal.

response to $\gamma$-rays was not altered by E4f1 overexpression.

Together, these results strongly suggest that $\mathrm{p} 16^{\mathrm{INK} 4 \mathrm{~A}} /$ $\mathrm{p} 19^{\mathrm{ARF}}$ and p53 are not required, at least separately, for the E4f1/Bmi1 genetic interaction.

E4f1 knockdown rescues Bmi1 ${ }^{-/-}$hematopoietic cells without affecting $\mathrm{p} 16^{\text {Inka }}$ and $\mathrm{p} 19^{\text {Arf }}$ expression

We next tested whether Bmil and E4f1 also interact genetically in primitive hematopoietic cells. Real-time Quantitative-PCR analysis demonstrated the coexpression of these two genes in the stem-cell-enriched subpopulations (KLS CD34- and KLS CD34 ${ }^{+}$) and confirmed the preferential expression of Bmil mRNA in cells with long-term repopulating activity (KLS CD34-) (Fig. 4A).

Flow cytometry analysis of E4F1 protein levels in fetal liver cells isolated from $\mathrm{Bmil}^{-/-}$or wild-type embryos revealed that a subpopulation of $\mathrm{Bmil}^{-/-}$cells expresses higher levels of E4F1 than populations of wild-type cells (Fig. 4B). Consistent with the high levels of Bmi1 expression in Scal-positive cells, we found that the accumulation of E4F1 occurs within the $\mathrm{Sca}^{+}$population of Bmil $^{-1-}$ cells (Fig. 4C).

Based on these observations and to further address the role of E4F1 in BMI-mediated HSCs proliferation, we examined whether E4f1 silencing could rescue the hematopoietic defect induced by loss of Bmil.

To test this possibility, fetal liver cells were isolated from $B \mathrm{mi1}^{-/}$or wild-type embryos and infected with shE4f1, Bmi1, or GFP (control) retroviruses as described in Figure 5A. Cultures initiated with wild-type $\left(\mathrm{Bmil}^{+/+}\right)$ cells infected with the control GFP vector generated nu- merous small and round cells with birefringent properties (Fig. 5B). In sharp contrast, $\mathrm{Bmil}^{-/}$cells infected with the control vector rapidly (by day 4) gave rise to poorly proliferating cultures that contained a majority of flat, granulous, and nonbirefringent cells (Fig. 5B). Qualitatively, the proliferation and morphological properties typical of $\mathrm{Bmi1}^{-/-}$cells were completely rescued by reducing the levels of E4f1 by RNA interference (Fig. 5B).

In agreement with these results, BrdU incorporation experiments indicated that while absence of Bmil resulted in a dramatic decrease in DNA synthesis /only $2.1 \% \mathrm{BrdU}^{+}$cells after $6 \mathrm{~d}$ of culture) and $\mathrm{G}_{1}$-phase accumulation (data not shown), reduction in E4F1 levels provided a rescue in DNA synthesis to levels comparable to those observed in wild-type cells (Fig. 5C). In agreement with findings in NIH $3 \mathrm{~T} 3$ cells, increasing levels of BMI1 resulted in enhanced BrdU incorporation (Fig. 5C). In addition, as shown by annexin $\mathrm{V}$ and propidium iodide staining, there was no significant difference in the proportion of cells undergoing apoptosis between wild-type or $\mathrm{Bmi1}^{-/-}$-derived cells (Fig. 5D), precluding a possible apoptotic effect of Bmil loss.

Since Bmil deletion in MEF induced a premature senescence phenotype associated with INK4A/ARF derepression, we asked whether $B \mathrm{mil}^{-/}$-derived hematopoietic cells also undergo cellular senescence as a consequence of $p 16^{\text {Ink } 4 a}$ and $p 19^{\text {Arf }}$ up-regulation. Therefore, cultures initiated with $\mathrm{Bmi1}^{-/}$cells were stained for senescence-associated $\beta$-galactosidase (SA- $\beta$-gal) activity, a well-established biomarker for senescent cells. While SA- $\beta$-gal activity is nearly undetectable in $\mathrm{Bmil}^{+/+}$-derived cultures $(<5 \%$ positive cells) (data not shown), the percentage of SA- $\beta-$ gal $^{+}$cells reached $70 \%-80 \%$ in cul- 
A

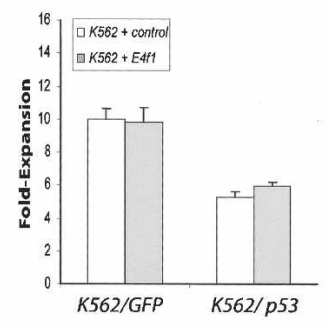

C

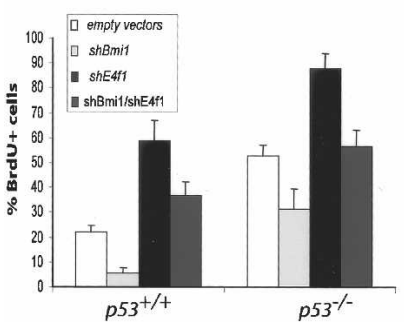

Figure 3. $\mathrm{p} 19^{\mathrm{Arf}} / \mathrm{p} 53$ is not required in Bmi1-E4f1 interaction. (A) $\mathrm{K} 562$ cells (INK4A/ $\mathrm{ARF}^{-/-} / \mathrm{p} 53^{-/-}$) were sequentially transduced with MSCV-p53-GFP (or appropriate empty vector) and $M S C V-N e o-E 4 f 1$ (or corresponding empty vector). Cells were kept in G418-containing medium during $7 \mathrm{~d}$ and then plated in triplicate and counted $4 \mathrm{~d}$ later. Relative cell expansions were measured in two independent experiments each resulting from newly infected and selected cellular populations assessed in triplicates within $6 \mathrm{~d}$ of infection. Data are given as means \pm SD. (B) NIH 3T3 cells (INK4A/ARF-/-/p53 ${ }^{+}$) were sequentially transduced with MSCV-E6- GFP (or appropriate empty vector) and $M S C V-N e o-E 4 f 1$ (or corresponding empty vector). Cells were kept in selective medium (containing G418 to prevent loss of E4f1 expressing retrovirus) during $7 \mathrm{~d}$ and then plated in triplicate and counted $4 \mathrm{~d}$ later. Relative proliferation rates were determined from two triplicate experiments (two independent infections). (C) BrdU incorporation assay in primary mouse em-

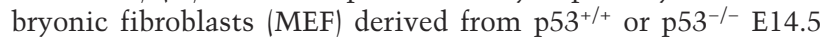
embryos and infected with combinations of control, shBmi1, and shE4f1 retroviruses. Cells were infected on day 3, and the BrdU pulse was $10 \mathrm{~h}$. BrdU incorporation on day 6 after infection was measured in two independent experiments each resulting from newly infected and selected cellular populations assessed in triplicates within $6 \mathrm{~d}$ of infection. Data are given as means $\pm \mathrm{SD}$. $(D)$ Whole cell protein extracts were prepared from control or E4f1-transduced NIH 3T3 cells at different time intervals (in hours) after exposure to a 5-Gy dose of ionizing radiation prior to Western blot analysis for E4F1, p21, and $\alpha$ TUBULIN (loading control).

tures initiated with GFP-transduced $\mathrm{Bmi1}^{-/-}$cells by day 7 (Fig. 5E, upper right panel). As described in previous work, we found that $\mathrm{p} 16^{\mathrm{INK} 4 \mathrm{~A}}$ and $\mathrm{p} 19^{\mathrm{ARF}}$ protein levels were significantly greater in GFP-transduced $\mathrm{Bmil}^{-/-}$ cells than in GFP-transduced wild-type cells (Fig. 5E, histogram overlay showing $16^{\mathrm{INK} 4 \mathrm{~A}}$ and p19 $9^{\mathrm{ARF}}$ levels, upper panels). The expression of mRNA levels for $p 16^{\text {Ink } 4 a}$ and $p 19^{A r f}$ in $B \mathrm{mil}^{-/-}$cells was also determined by quantitative RT-PCR (Q-PCR) and confirmed that $p 16^{\text {Ink } 4 a}$ and, to a lesser extent, $p 19^{A r f}$ mRNA levels were up- regulated on average 50 -fold and 20-fold compared to control wild-type cells after $7 \mathrm{~d}$ of culture, respectively (data not shown).

As expected, the reintroduction of $\mathrm{Bmil}$ in $\mathrm{Bmil}^{-/-}$ cells led to a nearly complete bypass of the senescence as determined by the decrease in the proportion of cells exhibiting SA- $\beta$-gal activity $\left(10 \%-20 \%\right.$ SA- $\beta$-gal ${ }^{+}$cells $)$ and down-regulation of $\mathrm{p} 16^{\mathrm{INK} 4 \mathrm{~A}}$ and $\mathrm{p} 19^{\mathrm{ARF}}$ proteins (Fig. 5E, middle panels) and mRNA (15-fold and 10-fold reduction) levels (data not shown).

Interestingly, $\mathrm{Bmi1}^{-/-}$cells engineered to express low E4F1 levels also escape (partially) senescence (30\%-40\% SA- $\beta$-gal ${ }^{+}$cells) but without affecting $\mathrm{p} 16^{\mathrm{INK} 4 \mathrm{~A}}$ or $\mathrm{p} 19^{\mathrm{ARF}}$ protein (Fig. 5E, lower panels) and mRNA levels (data not shown).

Reduction in E4F1 levels rescues proliferative defects of $\mathrm{Bmil}^{-1-}$ stem and progenitor cells

The in vitro and in vivo maintenance of progenitor and stem cell activity depends on the presence of Bmi1 (Lessard and Sauvageau 2003; Park et al. 2003). To determine whether $\mathrm{Bmil}^{-/-}$hematopoietic progenitor cells engineered to express low E4F1 levels could be recovered following in vitro cultures, we monitored colony-forming cell (CFC) activity at different time in cultures as indicated in Figure 5A. While at initiation of cultures (i.e., day 0) CFC activity was similar in all groups, their numbers increased steadily for $6 \mathrm{~d}$ in cultures initiated with either wild-type cells or with mutant cells engineered to express high levels of BMI1 (Fig. 5F). As expected, no progenitor activity could be recovered from cultures initiated with $\mathrm{Bmil}^{-/-}$cells transduced with GFP, but normal CFC activity was detected for $\mathrm{Bmil}^{-/-}$ cells in which levels of E4F1 were reduced (e.g., Fig. 5F, cf. bars and asterisk). These data indicate that E4f1 knockdown in Bmi1-deficient cells resulted in a complete rescue of their clonogenic activity. Importantly, E4f1 knockdown in wild-type cells had no effect on their capacity to generate CFCs, demonstrating the specificity of the reported effect (Fig. 5F).

In selected experiments, a competitive proliferation assay was set up by adjusting the proportion of transduced cells $\left(\mathrm{GFP}^{+}\right)$to $50 \%$ at initiation of the cultures. The evaluation of transduced CFCs along the culture showed that while wild-type cells transduced with GFP or shE4f1-GFP had no proliferative advantage over nontransduced cells, Bmi1-GFP-overexpressing cells expanded much better and became rapidly the predominant population of the culture (data not shown). Furthermore, after $4 \mathrm{~d}$ of culture, $>95 \%$ of CFCs generated from shE4f1- or Bmi1-transduced $\mathrm{Bmil}^{-/-}$cells were derived from transduced cells (i.e., $\mathrm{GFP}^{+}$), indicating a cell-autonomous nature for the rescue induced by E4f1 knockdown or Bmil complementation (data not shown).

In addition to severe proliferation defects, $\mathrm{Bmil}^{-/-}$progenitors maintained ex vivo for a short period of time became restricted in their differentiation potential to the monocyte/macrophage lineage. Reduction in E4F1 levels in these cells rescued their capacity for differentiation 
A
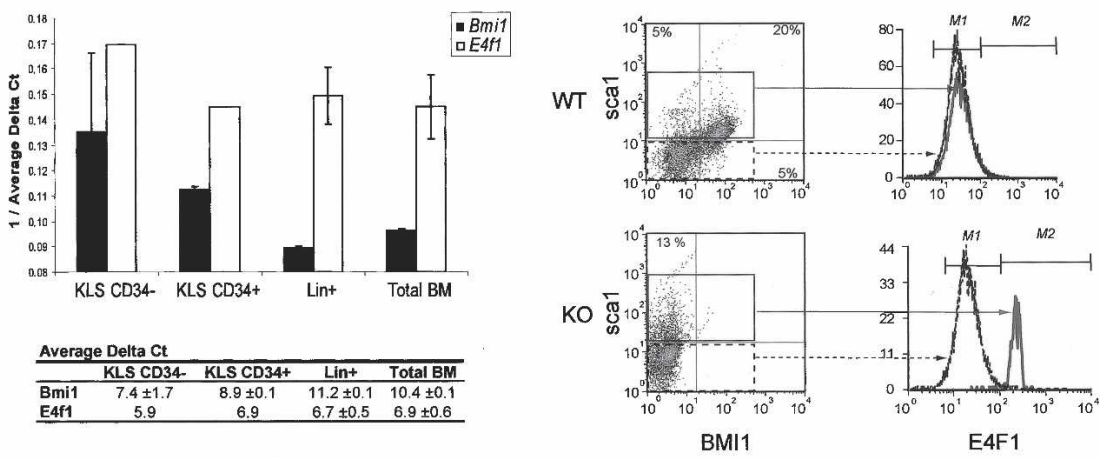

B
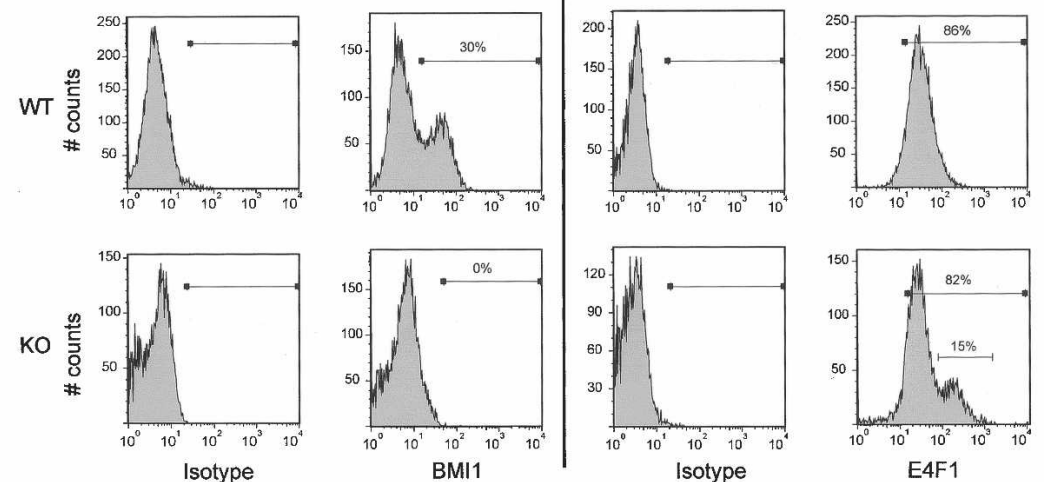

Figure 4. Expression of $E 4 f 1$ and Bmi1 in primitive hematopoietic cells. (A) Quantitative RT-PCR of Bmi1 and E4f1 in bone marrow (BM) hematopoietic cells: For each sorted cell population, mRNA levels were determined by calculating the $\Delta C t$ values where the levels of mRNA for $B m i 1$ and E4f1 are normalized according to the endogenous control gene Gapdh $\left(\Delta C t=C t_{\text {target }}-C t_{\text {endogenous control }}\right)$. The graph shows ratios of $1 / \Delta C t . \Delta C t$ values are shown in the table below. Experiments were repeated twice with bone marrow cells extracted from two series of $>150$ animals. Each Q-PCR reaction was performed in duplicate using automated pipette delivery systems (Biomek FX; Beckman). (B) $\mathrm{Bmil}^{+/+}$and $\mathrm{Bmi1}^{-/-}$fetal liver cells were cultured for $2 \mathrm{~d}$, fixed, permeabilized, and analyzed by flow cytometry for BMIl or E4F1 expression levels. (C) $\mathrm{Bmil}^{+/+}$and $\mathrm{Bmi1}^{-/-}$fetal liver cells were simultaneously stained with anti-Scal, anti-BMI1, and anti-E4F1 antibodies. The histogram overlay showed E4F1 expression levels in the Scal-negative population (hatched line) and the Sca1-positive population (red line). For $B$ and $C$, FACS profiles are representatives from more than three independent experiments. since no significant change in the proportion (or size) of multipotent (i.e., CFU-GEMM) or bipotent and unipotent (i.e., CFU-GM, CFU-G, or CFU-M) colonies was detected in 4-d cultures initiated with wild-type versus shE4f1-transduced Bmi1 ${ }^{-/-}$cells (Fig. 5G, left panel). Furthermore, the phenotypic characterization performed by flow cytometry confirmed the presence of primitive cells $\left(\mathrm{Lin}^{-} \mathrm{Scal}^{+}\right)$and mature cells $\left(\mathrm{Grl}^{+}\right.$or $\left.\mathrm{Macl}^{+}\right)$in the cultures derived from shE4f1-transduced cells (data not shown). In addition, examination of the Wright-stained cytospin preparations of cells recovered from these cultures (Fig. 5G, right panel) showed that only macrophages persisted in cultures derived from the control GFP-transduced $\mathrm{Bmil}^{-1-}$ cells, and that reduction of E4F1 protein levels in these cells enabled production of a variety of progenitors and differentiated cells.

Based on these observations, we presumed that the proliferative impairment of $\mathrm{Bmi1}^{-/-}$repopulating cells might be rescued by reducing E4F1 levels. To test this possibility, a series of recipients that received $\mathrm{Bmil}^{-/-}$ fetal liver cells transduced with shE4f1-GFP or with control GFP was generated. At multiple time points after transplantation, the recipients were analyzed for the presence of transduced donor cells $\left(\mathrm{GFP}^{+}\right.$cells) (Fig. 6A). In agreement with previous data, no donor-derived cells were detected in eight of eight recipients transplanted with GFP-transduced $\mathrm{Bmi1}^{-/-}$cells.

Interestingly, while E4f1 knockdown in wild-type cells does not lead to any detectable perturbation of hematopoietic repopulating ability, shE4f1-transduced
$B \mathrm{mi1}^{-/-}$cells showed an increased contribution to peripheral blood in the recipients tested, providing evidence for a strong genetic interaction between Bmi1 and $E 4 f 1$ in vivo for cells with repopulation potential lasting up to $12 \mathrm{wk}$ (Fig. 6A).

To determine whether engrafted shE4f1-GFP-transduced $B \mathrm{mi1}^{-/-}$cells also retained their full differentiation ability, peripheral blood cells were stained at $12 \mathrm{wk}$ post-transplantation for surface markers B220 (B-cell lineage), CD3 (T-cell lineage), Mac1 and Gr1 (myeloid lineage), in combination with the GFP marker. The presence of double-positive cells for GFP and each of these markers demonstrated the full potential of $\mathrm{Bmi1}^{-/-}$cells engineered to express low E4F1 levels to differentiate into B lymphoid (B220+), T lymphoid (CD3+), and myeloid cells (Mac1+ or Gr1+) (see Fig. 6B).

Although shE4f1 provides a good rescue of $\mathrm{Bmi1}^{-/-}$ cells with repopulation activity lasting at least $12 \mathrm{wk}$, it is important to analyze recipient mice at longer time points (e.g., >12-16 wk) because there is now clear evidence that at least two distinct and prospectively "isolable" populations of HSCs-termed short-term repopulating (STR) and long-term repopulating (LTR) HSCsare present in the mouse bone marrow (Zhao et al. 2000; Benveniste et al. 2003). When analyzed at 22 wk posttransplantation, the persistence of shE4f1-transduced $B \mathrm{mi1}^{-/-}$cells was observed at low level (i.e., $3 \% \pm 1 \%$ ) in the peripheral blood of three recipients out of four (Fig. 6C), indicating that STR-HSCs are rescued by shE4f1 but LTR-HSCs are not. 
Figure 5. Loss of $E 4 f 1$ rescues the proliferative defect of $B \mathrm{mil}^{-/-}$hematopoietic progenitors. (A) Overview of the experimental strategy: $\mathrm{Bmil}^{+/+}$and $\mathrm{Bmil}^{-/-}$fetal liver cells (the equivalent of one fetal liver) were infected for $2 \mathrm{~d}$ with GFP, Bmi1-GFP, or shE4f1-GFP retroviruses (at an efficiency of $80 \%-92 \%)$. The equivalent of one-half fetal liver was kept in culture for $10 \mathrm{~d}$ and assayed at different time points for their content in colony-forming cells (CFC). The equivalent of one-half fetal liver was transplanted in irradiated mice for in vivo study (see Fig. 6). (B) Bmi1 ${ }^{+/+}$ and $\mathrm{Bmil}^{-/-}$fetal liver cells engineered to express GFP or shE4f1-GFP retroviruses were visualized at day 4 of the culture under phase contrast microscopy (original magnification 20x). Representative of three independent experiments each with different fetal livers and retroviral preparations. $(C)$ BrdU incorporation assay in primary FL cells. Cells were pulsed with BrdU for $24 \mathrm{~h}$ on day 4 after infection, and incorporation was measured in three independent experiments each resulting from newly infected cellular populations assessed in duplicates. Data are given as means $\pm \mathrm{SD}$. $(D)$ The proportion of apoptotic cells was monitored by annexin V/PI staining. Representative of four independent experiments. (E) Bmi1/-/- fetal liver cells engineered to express GFP, Bmi1GFP, and shE4f1-GFP were assayed for senescence phenotype at day 6 of culture: The left panel showed representative flow cytometry analysis of $\mathrm{p} 16^{\text {Ink4a }}$ and $\mathrm{p} 19^{\text {Arf }}$ expression in transduced $B \mathrm{mi1}^{-/-}$cells (gray-filled histograms) compared to GFP-transduced wild-type cells (dotted line histogram), and the right panel shows a representative SA- $\beta$-gal staining of transduced $B \mathrm{mil}^{-/-}$cells. Representative of two independent experiments each performed in triplicate. $(F) B \mathrm{mi1}^{+/+}$and $B \mathrm{mi1}^{-/-}$fetal liver cells engineered to express GFP (ct, control), Bmi1-GFP, and shE4f1GFP were assayed for their content in colony-forming cells (CFC) at different time of the culture. Results show means \pm SD of four independent experiments performed in duplicate. $(G)$ Frequency of colony type was evaluated after $4 \mathrm{~d}$ of culture by morphological (in toto) analysis and Wright staining of representative colonies. Representative of four independent experiments performed in duplicate.

Interestingly the $\triangle \mathrm{HTH}$ mutant of BMIl (lacking interaction with E4F1) failed to restore both the short-term and long-term repopulating capacity of $\mathrm{Bmi1}^{-1-}$ cells, while excellent rescue was observed with wild-type BMI1 and the PEST deletion mutant, which preserves E4F1 interaction (Fig. 6C).

\section{Discussion}

In this study, we have identified E4F1 as a new binding partner of BMI1 and provide comprehensive evidence that this protein functionally interacts with BMI1 to regulate cell proliferation. This conclusion is supported by several lines of evidence: Firstly, we show that BMI1 and E4F1 interact physically and genetically both in cell lines (e.g., NIH 3T3) and in primary cells (MEF and hematopoietic cells) to control cell proliferation. Secondly, our data suggest that the physical interaction between these two proteins is important for this genetic interaction since the removal of the E4F1-interacting domain of Bmil eliminates its ability to rescue the proliferative defect induced by E4F1. Moreover, we provide evidence that INK4A/ARF and $p 53$ are independently not required for the genetic interaction between E4f1 and Bmi1 in selected cell lines and in MEFs. Finally and most importantly, the loss of E4F1 function in Bmi1 ${ }^{-/-}$hematopoietic cells leads to the rescue of their clonogenic and repopulating ability without affecting levels of $p 19^{A r f}$ or $p 16^{\text {Ink } 4 a}$, thus indicating that the effects of $E 4 f 1$ knockdown are not mediated through down-regulation of these CDK inhibitors.

Interestingly, the interaction between BMI1 and E4F1 appears to be restricted to the cytoplasm of dividing cells (J. Chagraoui, unpubl.), possibly revealing an unsuspected cytoplasmic and cell-cycle-dependent function for Bmil. This observation is consistent with previous 
A

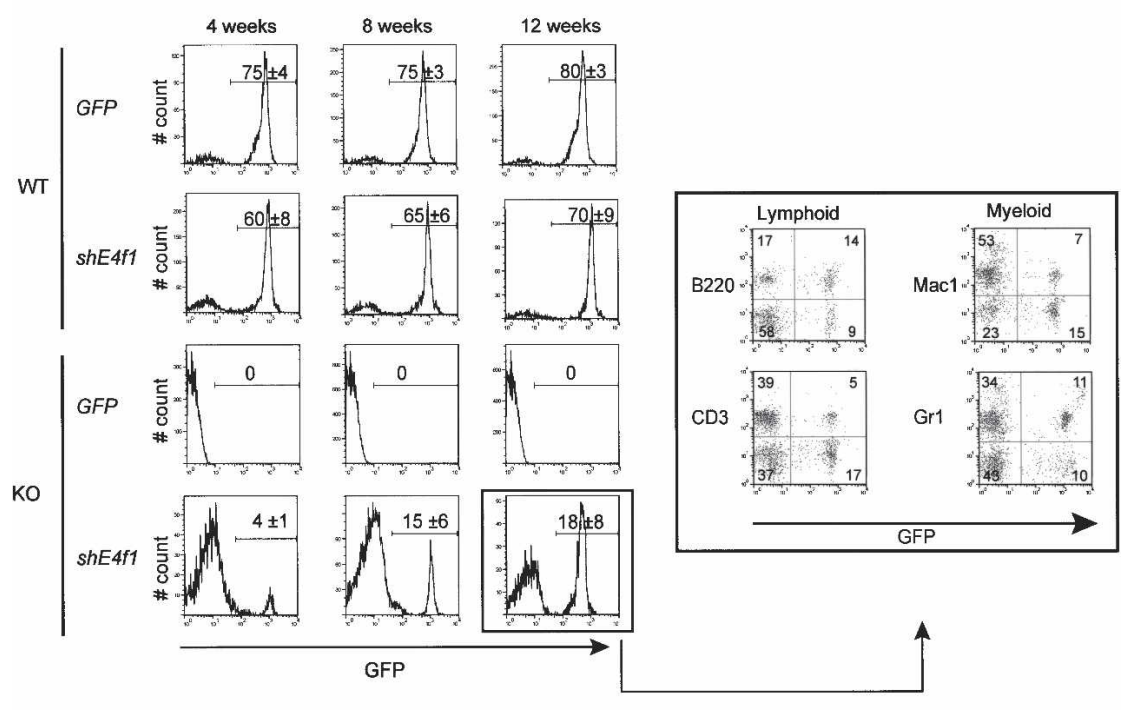

C

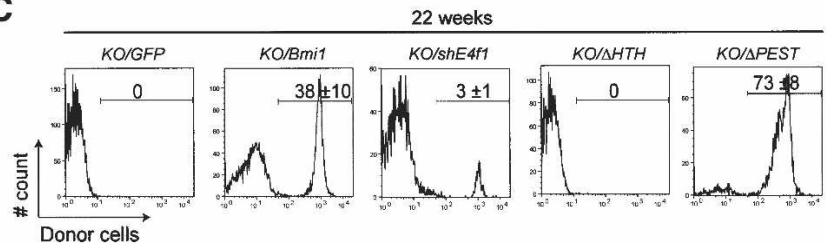

Figure 6. Loss of $E 4 f 1$ rescues the repopulating ability of Bmi1-deficient cells. (A) Two days after infection, $\mathrm{Bmi1}^{+/+}$and $\mathrm{Bmi1}^{-/-}$fetal liver (the equivalent of onehalf fetal liver) cells (Ly5.2) engineered to express GFP, Bmi1-GFP, or shE4f1-GFP were injected into lethally irradiated congenic (i.e., Ly5.1) recipient mice along with Ly5.1 competitor cells. Repopulation activity of the transduced cells was evaluated by monitoring donor cell chimerism $\left(\right.$ Ly5 $\left..2^{+} \mathrm{GFP}^{+}\right)$in peripheral blood 4,8 , and $12 \mathrm{wk}$ after transplantation. The figure shows representative FACS profiles from four recipients per group and $n=2$ independent experiments (mean values for repopulation $\pm \mathrm{SD}$ are indicated). $(B)$ Proportions of shE4f1-GFP transduced $\mathrm{Bmi1}^{-1-}$ cells in myeloid (Mac-1 and Gr1) and lymphoid (B220 and CD3) populations were determined at 12 wk posttransplantation. The data shown are representative FACS profiles from one recipient. Note that lympho-myeloid reconstitution was confirmed in all four mice analyzed (see $A) \cdot(C)$ Representative FACS profile showing donor contribution $\left(\mathrm{GFP}^{+}\right.$Ly $\left.5.2^{+}\right)$at 22 wk post-transplantation in the peripheral blood of recipients of $B \mathrm{mi1}^{-/-}$fetal liver cells engineered to express Bmi1-GFP, shE4f1-GFP, or Bmi1 mutants ( $\triangle \mathrm{HTH}$-GFP and $\triangle \mathrm{PEST}$-GFP). Note that recipients for shE4f1 transduced cells are the same as used in $A$ and $B$. Values are means \pm SD of six recipients per group (for Bmil and the indicated mutants).

data showing a dissociation of BMI1 from the chromatin during mitosis (Voncken et al. 1999; Voncken et al. 2005). Other emerging evidence also supports a role for PcG proteins in various cellular processes apart from chromatin modification. For example, EZH2 associates with VAV1 specifically in the cytoplasm and thus contributes in the control of actin polymerization and cellular proliferation (Nolz et al. 2005; Su et al. 2005). These findings raise the interesting prospect of two dynamic processes (an epigenetic and a nonepigenetic) involving BMI1 and will require further investigations. In regard to these observations, one possibility is that BMIl might sequester E4F1 or induce its degradation, thus counteracting its activity. This hypothesis, corroborated with the elevated E4F1 protein levels observed in the stemcell-enriched subpopulation (i.e., Scal ${ }^{+}$) of $\mathrm{Bmi1}^{-/-}$-deficient fetal liver cells, is further enforced by the new key role attributed to BMIl and its PcG partner RING1A in $\mathrm{H} 2 \mathrm{~A}$ ubiquitination as well as in their own ubiquitination (Cao et al. 2005; Buchwald et al. 2006).

Surprisingly, and although the literature points out $\mathrm{p} 19^{\mathrm{ARF}} / \mathrm{p} 53$ and $\mathrm{p} 16^{\mathrm{INK} 4 \mathrm{~A}} / \mathrm{Rb}$ as being the major effector pathways for E4F1 function, we found that $p 16^{\text {Ink } 4 a-/-}$ and $p 19^{A r f-I_{-}}$cell lines (NIH 3T3, U2OS) still undergo a cell cycle arrest on $E 4 f 1$ overexpression. In addition, the proliferative rescue obtained in $\mathrm{Bmil}^{-/-}$hematopoietic cells engineered to express a low level of E4F1 protein occurs without affecting $p 16^{I n k 4 a}$ and $p 19^{A r f}$ expression.
We also showed that the ability of p53 to induce p21 in response to irradiation is not affected by $E 4 f 1$ overexpression. Intriguingly, we did not observe a dramatic induction of p21 under E4f1 overexpression as it has been shown by Fernandes et al. (1998), although E4F1 protein levels appear higher in our E4f1-transduced NIH 3T3 cells than in the 3T3/E4f1 clone 7 (sent to us by Dr. R.J. Rooney and used as control) (data not shown). Moreover, in this clone 7 , we did not observe further induction of p21 after irradiation, suggesting a selective rather than a direct impact of E4F1 on p21 expression. Moreover, we show that $p 53$ deletion does not prevent the genetic interaction between E4f1 and Bmi1 in primary fibroblasts, although less pronounced than in wild type, indicating that p53 is not essential for the reported Bmi1/E4f1 genetic interaction. These data are further substantiated by the observation that $p 21^{-/-}$and $p 53^{-/-}$fibroblasts overexpressing $E 4 f 1$ are still blocked in $G_{1}$ phase (Fajas et al. 2000).

The conclusion that BMI1/E4F1 interplay occurs independently of INK4A/ARF and possibly p53 in selected cell lines does not exclude an important role for these genes in complementing the hematopoietic defects of Bmi1-deficient mice. Indeed, previous studies indicated that complete deletion of the INK4A/ARF locus and the disruption of the $p 53$ gene in Bmi1 mutant mice lead to a partial rescue of the hematopoietic defects (Bruggeman et al. 2005). It will thus be critical to investigate the genetic interaction between $E 4 f 1, I N K 4 A / A R F$, and $p 53$ 
in primitive hematopoietic cells. Such experiments will require the generation of $E 4 f 1$ conditional alleles. The inability of our E4f1 shRNA vector to confer a major rescue at 22 wk post-transplantation suggests that this gene is critical for the STR-HSCs and that LTR-HSCs may be rescued by inactivation of INK4A/ARF or $p 53$. Therefore, the molecular events downstream from BMI1 appear to be more complex than expected, and several plausible mechanisms might be involved: First, BMI1 represses INK4A/ARF transcription through a mechanism that remains to be fully characterized. Second, BMI may inhibit E4F1 activity (ubiquitination?) on several cell-cycle-regulating proteins, including the CDK inhibitor $\mathrm{p} 27^{\mathrm{KIP} 1}$, CYCLIN E, and CYCLIN B1, with reduced CDK2, CDK4/6, and CDC2 kinase activities and with the down-regulation of Cyclin A2 gene expression (Fernandes et al. 1998; Fajas et al. 2001; Rooney 2001). Moreover, HMGA2 protein, which plays an important role in chromatin organization, interacts with E4F1 and prevents its binding to the Cyclin A promoter (Tessari et al. 2003). Finally, E4F1 may participate in the mitotic spindle checkpoint by interacting with the tumor suppressor RASSF1A, known to control microtubule stability and cell cycle progression (Fenton et al. 2004; Ahmed-Choudhury et al. 2005). Consistent with these data, E4f1 is essential for embryonic development and cell division, as E4f1-deficient mouse embryos die at preimplantation stages and $E 4 f 1^{-/-}$ blastocysts display several mitotic abnormalities and chromosomal missegregation (Le Cam et al. 2004). These observations are most interesting since one of the eight BMI1interacting proteins identified in our yeast two-hybrid screen physically localizes to the centrosome and interacts with RASSF1A. It is thus conceivable that BMI1 may alter the interaction between E4F1 and microtubule-associated proteins to ensure normal chromosome segregation and cell cycle progression.

Together these observations suggest that BMI1 creates a complex but effective network for the control of cell proliferation and senescence and might orchestrate multiple pathways that cooperatively mediate E4F1 and INK4A/ARF function on the proliferation of stem and progenitor cells. Most interestingly, the analysis of the genes involved may clarify the molecular bases that underlie STR-HSCs versus LTR-HSCs.

\section{Materials and methods}

\section{Yeast two-hybrid screen}

A yeast two-hybrid screen was performed according to Clontech protocol using Bmi1 as bait to screen a human fetal liver cDNA library. Two rounds of screening yielded 14 positive clones corresponding to eight different cDNA sequences, all of which interacted specifically with BMIl but not with control baits (Large $\mathrm{T}$ antigen, LaminC). One of these cDNAs contained a 1.6-kb partial cDNA corresponding to $E 4 f 1$.

\section{Western blotting and immunoprecipitations}

Preparation of total, nuclear, and cytoplasmic extracts was done as described (Borden et al. 1998). Immunoprecipitations and immunoblots were done according to published methods.

\section{Quantitative RT-PCR}

The quantification of Bmi1 and E4f1 gene expression was performed in two independent experiments by real-time PCR on an ABI Prism 7900HT Sequence Detection System according to the manufacturer's recommendation. Briefly, KLS $\left(\mathrm{Kit}^{+} \mathrm{Lin}^{-} \mathrm{Sca}^{+}\right)$ $\mathrm{CD}_{4}^{-}{ }^{-}$, $\mathrm{KLS} \mathrm{CD} 4^{+}$, and $\mathrm{LIN}^{+}\left(\mathrm{B} 220^{+}\right.$; TER119 ${ }^{+}$; GR1 $\left.{ }^{+}\right)$subpopulations were sorted from two separate pools of mice bone marrow cells, and total RNA was harvested. For the total bone marrow (BM) sample, RNA was directly harvested from an aliquot of those pools. cDNA was generated using random primers and MMLV RT (Invitrogen) according to the manufacturer's protocol. Q-PCR reactions were carried out in a total volume of 10 $\mu \mathrm{L}$ on 25-50 ng of cDNA using TaqMan Universal Master Mix (Applied Biosystems) and Universal ProbeLibrary assays designed with the ProbeFinder software (Roche Applied Science). The primer sequences and Universal Probe numbers used are available upon request. ABI Prism 7900HT SDS was programmed to an initial step of $2 \mathrm{~min}$ at $50^{\circ} \mathrm{C}$ and $10 \mathrm{~min}$ at $95^{\circ} \mathrm{C}$, followed by 40 cycles of $15 \mathrm{sec}$ at $95^{\circ} \mathrm{C}$ and $1 \mathrm{~min}$ at $60^{\circ} \mathrm{C}$. All reactions were run in duplicate, and the average values were used for quantification. The mouse Gapdh predeveloped TaqMan assay was used as the endogenous control to normalize expression values of Bmi1 and E4f1 $\left(\Delta C T=C t_{\text {target }}-C t_{\text {Gapdh }}\right)$.

For $p 19^{I n k 4 a}$ and $p 16^{A r f}$ gene expression, total RNA was isolated by Trizol and DNase-I-treated, and cDNA was prepared according to the manufacturer's instructions (Invitrogen). Q-PCR reactions were carried out using TaqMan probe-based chemistry (Applera). The primer and probe sequences are as follows: p16 $6^{\text {Ink4a }}$, forward, 5'-CCCAACGCCCCGAACT-3', reverse, $5^{\prime}$-CGTGAACGTTGCCCATCA- $3^{\prime}$ and $5^{\prime}$-FAM-TCG GTCGTACCCCGATTCAGGTGAT-TAMRA-3'; p19 Arf, forward, 5'-GGGCCGCACCGGAAT-3', reverse, 5'-AGCAGAA

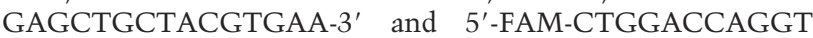
GATGATGATGGGCAAC-TAMRA-3'. The $18 \mathrm{~S}$ rRNA Taqman assay (ABI) was used as the endogenous control gene. Relative fold differences were determine by the $\Delta \Delta C t$ method using the wild-type fetal liver cells transduced with control GFP vector as the calibrator sample.

\section{Generation of shRNA}

Five short hairpin RNA (shRNA) sequences specific to E4f1 were designed and cloned in a retrovirus containing the RNA polymerase III promoter. Only one shRNA $\left(5^{\prime}\right.$-AAGATTCA GAAGACCTGCCAT-3') represses E4f1 efficiently. The shBmi1 encoding retrovirus was a gift from Martin Von Lohuizen (The Netherlands Cancer Institute).

\section{Mice genotyping}

Bmi1 mutant mice were genotyped by Southern blot analyses as described (van der Lugt et al. 1994). p53 mutant mice (Jackson Laboratory, Maine) were genotyped by PCR according to the manufacturer's instructions (Jax Mice Web site, http://jaxmice. jax.org).

\section{In vitro clonogenic progenitor assays}

For myeloid clonogenic progenitor assays, cells were plated in $35-\mathrm{mm}$ dishes in semisolid medium, containing $1 \%$ methylcellulose in $\alpha$ medium supplemented with $10 \%$ fetal calf serum (FCS), $5.7 \%$ bovine serum albumin, $10^{-5} \beta$-mercaptoethanol ( $\beta$ $\mathrm{ME}), 1 \mathrm{U} / \mathrm{mL}$ erythropoietin (Epo), IL-3, IL-6, Steel, $2 \mathrm{mM}$ glutamine, and $200 \mathrm{mg} / \mathrm{mL}$ transferrin. Colonies were scored on day 7 of incubation as derived from CFU-GM, BFU-E, or CFUGEMM according to standard criteria. For some of the experi- 
ments, identification of the colony types was confirmed by Wright staining of cytospins preparations of colonies.

Retroviral generation, infection, and transplantation of E14.5 fetal liver and bone marrow cells

Details of the different retroviral vectors used in this study are available on request. Production of vesicular stomatitis viruspseudotyped (VSV) retroviruses, infection of hematopoietic cells, and transplantation into mice were done as described (Thorsteinsdottir et al. 2002).

\section{Flow cytometry}

Flow cytometry analyses were performed using a LSRII cytometer (Becton Dickinson). For intracellular antigens, cells were first fixed with $3.7 \%$ paraformaldehyde and permeabilized with $0.25 \%$ Triton X-100. BMI1 and E4F1 were revealed using primary antibodies (monoclonal anti-Bmil antibody [Clone F6, Upstate] and polyclonal rabbit anti-E4F1 antibody [provided by R.J. Rooney], respectively) and secondary antibodies (goat antimouse PE, goat anti-rabbit FITC).

\section{Cell cycle and apoptosis analyses}

Analysis of BrdU incorporation was performed using the BrdU Flow Kit (PharMingen) in accordance with the manufacturer's instructions. Cell cycle analysis was performed after ethanol fixation, RNase digestion, and propidium iodide staining using BD ModFit LT software. Cultured cells from $\mathrm{Bmi1}^{-/-}$or $\mathrm{Bmi1}^{+/+}$ fetal livers were stained with FITC-annexin V (Becton Dickinson) and propidium iodide $(50 \mu \mathrm{g} / \mathrm{mL})$ in accordance with the manufacturer's instructions, and analyzed by flow cytometry.

\section{Senescence analyses}

SA- $\beta$-galactosidase activity was determined using a SA- $\beta$-gal staining kit from Cell Signaling Technology according to the manufacturer's instructions. Briefly, hematopoietic cells harvested from 7 -d fetal liver cultures were seeded onto polylysinecoated slides. Cells were fixed in $2 \%(\mathrm{v} / \mathrm{v})$ formaldehyde and $0.2 \%$ glutaraldehyde, and then incubated in SA- $\beta$-gal staining solution $(1 \mathrm{mg} / \mathrm{mL}$ 5-bromo-4-chloro-3-indolyl $\beta$-D-galactosidase; $40 \mathrm{mM}$ citric acid at $\mathrm{pH} 6.0,40 \mathrm{mM}$ sodium phosphate at $\mathrm{pH}$ 6.0, $5 \mathrm{mM}$ potassium ferrocyanide, $5 \mathrm{mM}$ potassium ferricyanide, $150 \mathrm{mM}$ sodium chloride, and $2 \mathrm{mM}$ magnesium chloride) at $37^{\circ} \mathrm{C}$ for $10 \mathrm{~h}$. Senescent cells were identified as bluestained cells by standard light microscopy, and a total of 500 cells were counted in five random fields on a slide to determine the percentage of SA- $\beta$-gal-positive cells.

\section{Acknowledgments}

We acknowledge R.J. Rooney for providing critical material for these experiments, in particular, the antiserum to E4F1, the expression vector, and the NIH 3T3 clones engineered to overexpress E4F1. We also acknowledge Melanie Frechette for her expertise and help with the maintenance and manipulation of the animals, Danièle Gagné for assistance with flow cytometry and cell sorting, and Christian Charbonneau for assistance in figures and photography. We thank Sonia Cellot, Jana Krosl, and Amelie Faubert for discussions and critical comments on the manuscript. This work was supported by grants from the CIHR to G.S. and S.M. G.S. and S.M. hold Canada Research Chairs in Molecular Genetics of Stem Cells and Cellular Signaling, respectively. G.S. is a scholar of the Leukemia Lymphoma Society of America.

\section{References}

Ahmed-Choudhury, J., Agathanggelou, A., Fenton, S.L., Ricketts, C., Clark, G.J., Maher, E.R., and Latif, F. 2005. Transcriptional regulation of cyclin A2 by RASSF1A through the enhanced binding of $\mathrm{p} 120^{\mathrm{E} 4 \mathrm{~F}}$ to the cyclin $\mathrm{A} 2$ promoter. Cancer Res. 65: 2690-2697.

Benveniste, P., Cantin, C., Hyam, D., and Iscove, N.N. 2003. Hematopoietic stem cells engraft in mice with absolute efficiency. Nat. Immunol. 4: 708-713.

Borden, K.L., CampbellDwyer, E.J., Carlile, G.W., Djavani, M., and Salvato, M.S. 1998. Two RING finger proteins, the oncoprotein PML and the arenavirus $\mathrm{Z}$ protein, colocalize with the nuclear fraction of the ribosomal P proteins. J. Virol. 72: 3819-3826.

Bruggeman, S.W., Valk-Lingbeek, M.E., van der Stoop, P.P., Jacobs, J.J., Kieboom, K., Tanger, E., Hulsman, D., Leung, C., Arsenijevic, Y., Marino, S., et al. 2005. Ink4a and Arf differentially affect cell proliferation and neural stem cell selfrenewal in Bmil-deficient mice. Genes \& Dev. 19: 14381443.

Buchwald, G., van der Stoop, P., Weichenrieder, O., Perrakis, A., van Lohuizen, M., and Sixma, T.K. 2006. Structure and E3ligase activity of the Ring-Ring complex of Polycomb proteins Bmil and Ring1b. EMBO T. 25: 2465-2474.

Cao, R., Tsukada, Y., and Zhang, Y. 2005. Role of Bmi-1 and Ring1A in H2A ubiquitylation and Hox gene silencing. Mol. Cell 20: 845-854.

Core, N., Bel, S., Gaunt, S.J., Aurrand-Lions, M., Pearce, J., Fisher, A., and Diabali, M. 1997. Altered cellular proliferation and mesoderm patterning in Polycomb-M33-deficient mice. Development 124: 721-729.

Fajas, L., Paul, C., Zugasti, O., Le Cam, L., Polanowska, J., Fabbrizio, E., Medema, R., Vignais, M.L., and Sardet, C. 2000. pRB binds to and modulates the transrepressing activity of the E1A-regulated transcription factor $\mathrm{p} 120^{\mathrm{E} 4 \mathrm{~F}}$. Proc. Natl. Acad. Sci. 97: 7738-7743.

Fajas, L., Paul, C., Vie, A., Estrach, S., Medema, R., Blanchard, J.M., Sardet, C., and Vignais, M.L. 2001. Cyclin A is a mediator of p120 $24 \mathrm{~F}$-dependent cell cycle arrest in $\mathrm{G}_{1}$. Mol. Cell. Biol. 21: 2956-2966.

Fenton, S.L., Dallol, A., Agathanggelou, A., Hesson, L., AhmedChoudhury, J., Baksh, S., Sardet, C., Dammann, R., Minna, J.D., Downward, J., et al. 2004. Identification of the E1Aregulated transcription factor $\mathrm{p} 120^{\mathrm{E} 4 \mathrm{~F}}$ as an interacting partner of the RASSF1A candidate tumor suppressor gene. Cancer Res. 64: 102-107.

Fernandes, E.R., Zhang, J.Y., and Rooney, R.J. 1998. Adenovirus E1A-regulated transcription factor $\mathrm{p} 120^{\mathrm{E} 4 \mathrm{~F}}$ inhibits cell growth and induces the stabilization of the cdk inhibitor p21 WAF1. Mol. Cell. Biol. 18: 459-467.

Hernandez-Munoz, I., Lund, A.H., van der Stoop, P., Boutsma, E., Muijrers, I., Verhoeven, E., Nusinow, D.A., Panning, B., Marahrens, Y., and van Lohuizen, M. 2005. Stable X chromosome inactivation involves the PRC1 Polycomb complex and requires histone MACROH2A1 and the CULLIN3/ SPOP ubiquitin E3 ligase. Proc. Natl. Acad. Sci. 102: 76357640.

Itahana, K., Zou, Y., Itahana, Y., Martinez, J.L., Beausejour, C., Jacobs, J.J., Van Lohuizen, M., Band, V., Campisi, J., and Dimri, G.P. 2003. Control of the replicative life span of human fibroblasts by p16 and the polycomb protein Bmi-1. Mol. Cell. Biol. 23: 389-401.

Iwama, A., Oguro, H., Negishi, M., Kato, Y., Morita, Y., Tsukui, H., Ema, H., Kamijo, T., Katoh-Fukui, Y., Koseki, H., et al. 2004. Enhanced self-renewal of hematopoietic stem cells 
mediated by the Polycomb gene product Bmi-1. Immunity 21: 843-851.

Jacobs, J.J., Kieboom, K., Marino, S., DePinho, R.A., and van Lohuizen, M. 1999. The oncogene and Polycomb-group gene bmi-1 regulates cell proliferation and senescence through the ink4a locus. Nature 397: 164-168.

Kamijo, T., Weber, J.D., Zambetti, G., Zindy, F., Roussel, M.F., and Sherr, C.J. 1998. Functional and physical interactions of the ARF tumor suppressor with p53 and Mdm2. Proc. Nat1. Acad. Sci. 95: 8292-8297.

Le Cam, L., Lacroix, M., Ciemerych, M.A., Sardet, C., and Sicinski, P. 2004. The E4F protein is required for mitotic progression during embryonic cell cycles. Mol. Cell. Biol. 24: 64676475.

Lessard, J. and Sauvageau, G. 2003. Bmi-1 determines the proliferative capacity of normal and leukaemic stem cells. $\mathrm{Na}$ ture 423: 255-260.

Lessard, J., Baban, S., and Sauvageau, G. 1998. Stage-specific expression of Polycomb group genes in human bone marrow cells. Blood 91: 1216-1224.

Lessard, J., Schumacher, A., Thorsteinsdottir, U., van Lohuizen, M., Magnuson, T., and Sauvageau, G. 1999. Functional antagonism of the Polycomb-Group genes eed and Bmil in hemopoietic cell proliferation. Genes \& Dev. 13: 2691-2703.

Miyazaki, M., Kawamoto, H., Kato, Y., Itoi, M., Miyazaki, K., Masuda, K., Tashiro, S., Ishihara, H., Igarashi, K., Amagai, T., et al. 2005. Polycomb group gene mel-18 regulates early $\mathrm{T}$ progenitor expansion by maintaining the expression of Hes1, a target of the Notch pathway. I. Immunol. 174: 25072516.

Moehrle, A. and Paro, R. 1994. Spreading the silence: Epigenetic transcriptional regulation during Drosophila development. Dev. Genet. 15: 478-484.

Molofsky, A.V., He, S., Bydon, M., Morrison, S.J., and Pardal, R. 2005. Bmi-1 promotes neural stem cell self-renewal and neural development but not mouse growth and survival by repressing the $\mathrm{p} 16^{\mathrm{Ink} 4 \mathrm{a}}$ and $\mathrm{p} 19^{\text {Arf }}$ senescence pathways. Genes \& Dev. 19: 1432-1437.

Nolz, J.C., Gomez, T.S., and Billadeau, D.D. 2005. The Ezh2 methyltransferase complex: Actin up in the cytosol. Trends Cell Biol. 15: 514-517.

Ohta, H., Sawada, A., Kim, J.Y., Tokimasa, S., Nishiguchi, S., Humphries, R.K., Hara, J., and Takihara, Y. 2002. Polycomb group gene rae 28 is required for sustaining activity of hematopoietic stem cells. J. Exp. Med. 195: 759-770.

Park, I.K., Qian, D., Kiel, M., Becker, M.W., Pihalja, M., Weissman, I.L., Morrison, S.J., and Clarke, M.F. 2003. Bmi-1 is required for maintenance of adult self-renewing haematopoietic stem cells. Nature 423: 302-305.

Pomerantz, J., Schreiber-Agus, N., Liegeois, N.J., Silverman, A., Alland, L., Chin, L., Potes, J., Chen, K., Orlow, I., Lee, H.W., et al. 1998. The Ink4a tumor suppressor gene product, p19 ${ }^{\text {Arf }}$, interacts with MDM2 and neutralizes MDM2's inhibition of p53. Cell 92: 713-723.

Quelle, D.E., Zindy, F., Ashmun, R.A., and Sherr, C.J. 1995. Alternative reading frames of the INK4a tumor suppressor gene encode two unrelated proteins capable of inducing cell cycle arrest. Cell 83: 993-1000.

Rooney, R.J. 2001. Cell cycle attenuation by $\mathrm{p} 120^{\mathrm{E} 4 \mathrm{~F}}$ is accompanied by increased mitotic dysfunction. Cell Growth Differ. 12: 505-516.

Serrano, M., Hannon, G.J., and Beach, D. 1993. A new regulatory motif in cell-cycle control causing specific inhibition of cyclin D/CDK4. Nature 366: 704-707.

Su, I.H., Dobenecker, M.W., Dickinson, E., Oser, M., Basavaraj, A., Marqueron, R., Viale, A., Reinberg, D., Wulfing, C., and
Tarakhovsky, A. 2005. Polycomb group protein ezh2 controls actin polymerization and cell signaling. Cell 121: 425436.

Tessari, M.A., Gostissa, M., Altamura, S., Sgarra, R., Rustighi, A., Salvagno, C., Caretti, G., Imbriano, C., Mantovani, R., Del Sal, G., et al. 2003. Transcriptional activation of the cyclin A gene by the architectural transcription factor HMGA2. Mol. Cell. Biol. 23: 9104-9116.

Thorsteinsdottir, U., Mamo, A., Kroon, E., Jerome, L., Bijl, J., Lawrence, H.J., Humphries, K., and Sauvageau, G. 2002. Overexpression of the myeloid leukemia-associated Hoxa9 gene in bone marrow cells induces stem cell expansion. Blood 99: 121-129.

Tokimasa, S., Ohta, H., Sawada, A., Matsuda, Y., Kim, J.Y., Nishiguchi, S., Hara, J., and Takihara, Y. 2001. Lack of the Polycomb-group gene rae 28 causes maturation arrest at the early B-cell developmental stage. Exp. Hematol. 29: 93-103.

Valk-Lingbeek, M.E., Bruggeman, S.W., and van Lohuizen, M. 2004. Stem cells and cancer; the Polycomb connection. Cell 118: 409-418.

van der Lugt, N.M., Domen, J., Linders, K., van Roon, M., Robanus-Maandag, E., te Riele, H., van der Valk, M., Deschamps, J., Sofroniew, M., van Lohuizen, M., et al. 1994. Posterior transformation, neurological abnormalities, and severe hematopoietic defects in mice with a targeted deletion of the bmi-1 proto-oncogene. Genes \& Dev. 8: 757-769.

Voncken, J.W., Schweizer, D., Aagaard, L., Sattler, L., Jantsch, M.F., and van Lohuizen, M. 1999. Chromatin-association of the Polycomb group protein BMI1 is cell cycle-regulated and correlates with its phosphorylation status. J. Cell Sci. 112: 4627-4639.

Voncken, J.W., Niessen, H., Neufeld, B., Rennefahrt, U., Dahlmans, V., Kubben, N., Holzer, B., Ludwig, S., and Rapp, U.R. 2005. MAPKAP kinase $3 \mathrm{pK}$ phosphorylates and regulates chromatin association of the Polycomb group protein Bmil. J. Biol. Chem. 280: 5178-5187.

Wang, H., Wang, L., Erdjument-Bromage, H., Vidal, M., Tempst, P., Jones, R.S., and Zhang, Y. 2004. Role of histone H2A ubiquitination in Polycomb silencing. Nature 431: 873-878.

Weber, J.D., Taylor, L.J., Roussel, M.F., Sherr, C.J., and Bar-Sagi, D. 1999. Nucleolar Arf sequesters Mdm2 and activates p53. Nat. Cell Biol. 1: 20-26.

Zhao, Y., Lin, Y., Zhan, Y., Yang, G., Louie, J., Harrison, D.E., and Anderson, W.F. 2000. Murine hematopoietic stem cell characterization and its regulation in BM transplantation. Blood 96: 3016-3022. 